\title{
Special issue on device-free sensing for human behavior recognition
}

\author{
Bin Guo ${ }^{1}$. Yanyong Zhang ${ }^{2} \cdot$ Daqing Zhang ${ }^{3} \cdot$ Zhu Wang $^{1}$ \\ Published online: 25 January 2019 \\ (C) Springer-Verlag London Ltd., part of Springer Nature 2019
}

We are delighted to present this special issue of Personal and Ubiquitous Computing on Device-Free Sensing for Human Behavior Recognition. Recently, device-free human behavior recognition approaches (e.g., Wi-Fi based and acoustic based) are attracting increasing attentions. For instance, compared with traditional approaches, Wi-Fi based approaches have a set of advantages, e.g., they do not require lighting, provide better coverage as they can operate through walls, preserve user privacy, and do not require users to carry any devices as they rely on Wi-Fi signals reflected by humans. As a result, the recognition of quite a number of behaviors that are difficult based on traditional approaches have now become possible, e.g., fine-grained movements (e.g., gesture and lip language), keystrokes, drawings, gait patterns, vital signals (e.g., breathing rate and heart rate), etc.

The aim of this special issue is to investigate the recent development of new frameworks, mechanisms, and algorithms that are able to support device-free behavior recognition systems. Following an open call for papers, we received a

Zhu Wang

wangzhu@nwpu.edu.cn

Bin Guo

guob@nwpu.edu.cn

Yanyong Zhang

yyzhang@winlab.rutgers.edu

Daqing Zhang

dqzhang@sei.pku.edu.cn

1 School of Computer Science, Northwestern Polytechnical University, Xi'an 710072, China

2 Department of Electrical \& Computer Engineering, Rutgers University, North Brunswick, NJ 08902-3390, USA

3 Software Engineering Institute, Peking University, Beijing 100871, China total number of 15 submissions. All the submissions have undergone rigorous peer-review according to the journal's high standards. Based on recommendations from the reviewers, the guest editors selected 5 contributions (with a accept rate of $33 \%$ ) which cover different topics within this theme, ranging from device-free localization to gesture recognition, from activity tracking to behavior profiling.

The first article, by Wang et al. on "DTransfer: extremely low cost localization irrelevant to targets and regions for activity recognition" proposes an low-cost device-free localization approach that is able to localize different kinds of targets in different regions. In particular, an optimized low-rank matrix completion model is adopted to construct the sensing matrix (i.e., radio-map) of the original region to reduce the overhead, and a rigorously designed quadratic transfer scheme is proposed to accurately locate different categories of targets in different regions.

In "Dynamic gesture recognition using wireless signals with less disturbance", the authors develop a gesture recognition system by exploring fine-grained Wi-Fi Channel State Information (CSI). To address the challenge that moving objects may have significant disturbance to wireless signal-based gesture recognition, a disturbance eliminating mechanism is developed for disturbance elimination.

Wang et al. in "Enabling non-invasive and real-time human-machine interactions based on wireless sensing and fog computing" propose a novel gesture recognition system by integrating the advantages of Doppler radar-based wireless sensing and fog computing, which is able to facilitate noninvasive and real-time human-machine interactions. In particular, a threshold detection method is adopted to extract gesture segments, and a two-stage classification method is proposed to recognize human gestures.

In "Automated employee evaluation using fuzzy and neural network synergism through IoT assistance", to make the evaluation of employee solely performance oriented, the authors propose a neuro-fuzzy-based framework by leveraging wireless sensing. Specifically, for detecting and tracking the 
employee activities, Internet of things (IoT)-enabled sensors are used, while artificial neural fuzzy inference system (ANFIS) is used for learning and automated decision optimization.

Chen et al. in "Trip2Vec: a deep embedding approach for clustering and profiling taxi trip purposes" propose a devicefree and novel behavior profiling model. Taxi trip related context information are extracted as the clue of behavior purposes, and a deep embedding approach is developed to get a more semantical and discriminative context representation in the latent space. Moreover, trips with similar latent representation are aggregated, and based the aggregation results behavior purposes can be understood in the city-wide level.

The guest editors believe the papers appearing in this issue form an accurate representation of current research topics in device-free sensing for human behavior recognition, and hope these articles will stimulate further development in this area. The guest editors express their appreciation to the authors and reviewers for contributing to this special issue. We also would like to express our appreciation to the editor in chief and the staff of the Personal and Ubiquitous Computing Journal for their kind help in accomplishing this special issue.

We hope you enjoy this special issue and take some inspiration from it for your own future research.

Publisher's note Springer Nature remains neutral with regard to jurisdictional claims in published maps and institutional affiliations. 